\title{
How the Analyzer can Help the User Help the Analyzer
}

\author{
Yannick Moy \\ AdaCore \\ moy@adacore.com
}

\begin{abstract}
The automation offered by modern program proof tools goes hand in hand with the capability to interact with the tool when the verification fails. The SPARK proof tool tries to help the user by providing the right information, so that the user can help the tool complete the proof. In this article, we present these mechanisms and how they work concretely on a simple running example.
\end{abstract}

\section{Introduction}

Program proof is the application of deductive verification techniques to programs. Industrial acceptability of such tools relies on the high degree of automation provided by modern automatic provers, in particular SMT solvers, when the source code is restricted to a suitable language subset. SPARK ${ }^{1}$ is an example of such a FLOSS industrial tool for Ada programs. It is available freely online as part of GNAT Community Edition 2

While striving to offer the most automation to our users, we have also recognized early on the need for interactions when the analyzer cannot complete the verification automatically. In those cases, the task for the analyzer is to display to the user the right information that will allow her to provide in exchange the pieces of information that are required for the analyzer to complete the verification.

Over the years, we have come up with a variety of solutions to address this challenge, trying to adapt these solutions to the degree of expertise of the user. All these solutions came from discussions (usually by email) with industrial users of SPARK, as part of the support activity that they subscribed to: a user asks about a puzzling message or a problem with proving a property; we explain the possible problem and show how this can be investigated; through discussion we together come up with a way to include part of this explanation in the messages of the analyzer. We have lots of anecdotal evidence that this helps users, in the form of positive feedback to our message improvements. We are presenting these solutions in this article, in the hope that they can be useful to others, and serve as a basis for better future solutions, as the challenges presented here are common to most similar analyzers.

\section{The Nurse: Providing First Aid}

At a minimum, the analyzer should help the user understand what is the problem, using in particular clear messages with precise locations. This is not always enough, due to the complexity of the detailed program semantics at play which may elude the user. This difficulty is compounded by the lack of expertise for novice users of the programming language. The solution we have adopted in SPARK is to augment the basic message with additional information explaining the immediate cause of the problem. Let's consider a procedure Erase which replaces every character in a string with the blank character:

$1 \longdiv { \text { https: //www.adacore.com/sparkpro } }$

2 https://www.adacore.com/download

A. Paskevich, J. Proença (Eds.): Formal Integrated Development Environment 2021 (F-IDE 2021)

EPTCS 338, 2021, pp. 97-104 doi 10.4204/EPTCS.338.12
(C) Y. Moy

This work is licensed under the Creative Commons Attribution License. 


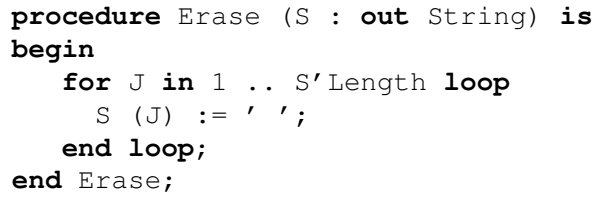

When running GNATprove (the name of the analysis tool for SPARK) on that code, it issues the following message:

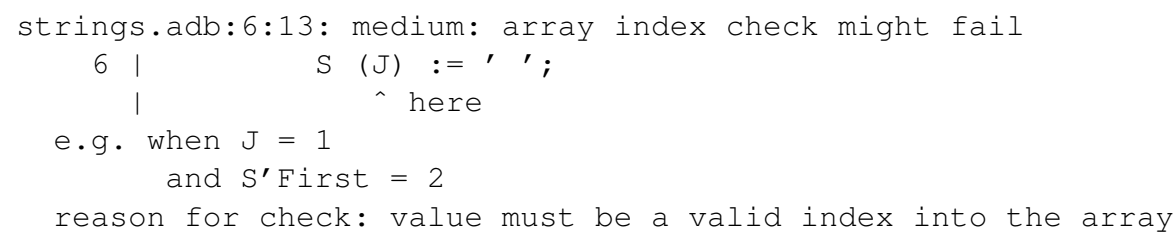

The main message line contains the precise location of the message, its severity, and the message text indicating which property might be violated. Here, this is an array index check, which corresponds to the run-time check when accessing an array in SPARK. The next two lines are only present in command-line usage, and help visually locate the issue outside of an IDE. So far, this is what every tool diagnostic should contain.

The purpose of the remaining lines is to help the user understand the reason for the message to be issued. The counterexample introduced by "e.g." gives concrete values of variables which lead to a property violation. Here, when $\mathrm{J}$ is one and the string $\mathrm{S}$ starts at index two (denoted in SPARK by $S^{\prime}$ First), the assignment attempts to write outside of the bounds of the array. This is reinforced by the last line, which gives the reason for a check at this location: assigning into an array in SPARK requires the index to be within bounds.

These messages are particularly useful for beginners, who may not know that arrays (and among them, strings) in SPARK may start at other indexes than one, or who may not realize that assigning into an array involves implicitly a run-time check that this assignment is within bounds.

The "reason for the check" provides additional context which can be useful also to more advanced users, in cases where the details of the language semantics requiring a check are more complex. It also facilitates understanding exactly in which part of a longer expression a check originates, as there might be many similar checks on the same line.

Only part of the counterexample is displayed above as part of the message. A counterexample is really a trace inside the subprogram consisting in multiple program points with values of variables at each program point. In the GNAT Studio IDE, the user can choose to display the trace with a simple click on a magnify icon next to the message.

Still in this category of first-aid help, GNATprove strives to present the user with the smallest subproperty that cannot be proved, in cases where the property to prove is a conjunction of smaller subproperties or a universally quantified property. This is particularly useful when attempting to prove the precondition of a call or a loop invariant, which typically are a conjunction of sub-properties. This requires GNATprove to split unproved properties further until a leaf sub-property is not proved.

\section{The Investigator: Looking for Probable Cause}

Once the user understands the immediate cause of the problem, the next step is to understand its root cause. Similar to the use of backtrace when debugging a program, the analyzer should provide information on the context of the problem that helps identify the missing link in the chain of deductions that the 
user does in her head. In particular, programmers rely on operational semantics to understand program executions, while the analyzer relies on axiomatic semantics which may abstract away crucial details of the operational semantics. The solution we have adopted in SPARK is to output additional information related to the problem when there is a chance that it might have been overlooked by the user. Let's fix our implementation of Erase by iterating over the range of string $\mathrm{S}$ :

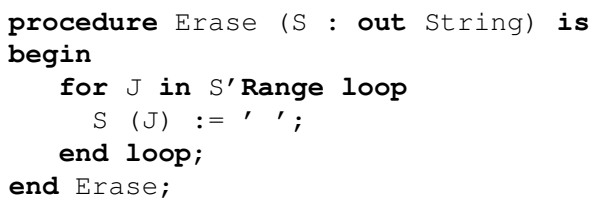

and let's add a contract to Erase specifying in a postcondition that all characters in $\mathrm{s}$ should be blank on return:

procedure Erase (S : out String)

with Post $\Rightarrow$ All_Blanks (S);

which is defined recursively over the range of $s$ :

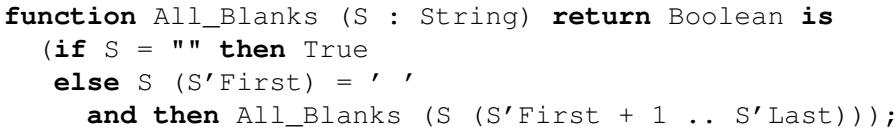

When running GNATprove on that code, it issues the following messages:

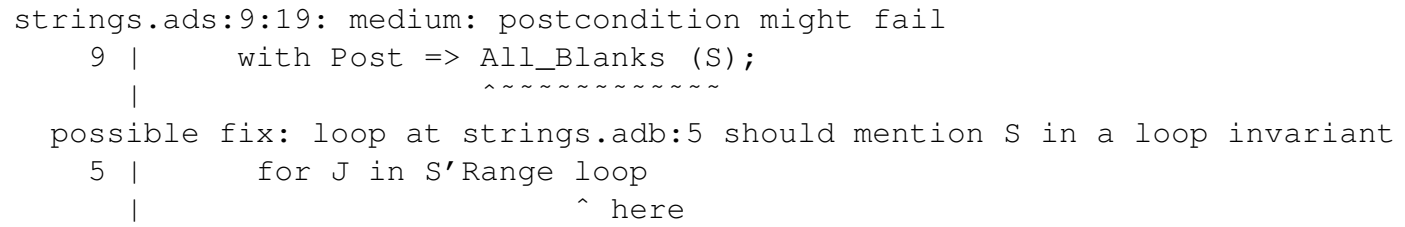

The three last lines are new compared to the messages in the previous section. GNATprove points here to a possible cause for the failure to prove the postcondition, which is that the loop in Erase has no loop invariant. It comes to this conclusion by looking at the variables which are mentioned (explicitly or implicitly) in the property to prove, here only s, and traverses the code in reverse execution order from the program point where the property should hold. During this traversal, it correctly identifies here that the loop modifies $\mathrm{S}$ without specifying how those changes impact the value of $\mathrm{S}$ inside a loop invariant, which is a likely cause for not being able to prove the postcondition.

A common pitfall of program proof is the frame problem. For automatic provers to be able to reason about formulas that represent the program semantics, these formulas necessarily must encode small parts of the whole program semantics. Thus GNATprove defines a frame for each property to check, that only presents a subset of the information available in the program, abstracting in particular subprogram calls as the corresponding subprogram contract and loop iterations as the loop invariant for that loop. When the user did not write a contract for a subprogram, or did not write a loop invariant for a loop, GNATprove may still be able to analyze the corresponding call/loop precisely by inlining the call or unrolling the loop. But this is not always the case, which raises the question of how these internal tool decisions are communicated to the user. Because this kind of information was a source of confusion for beginners, GNATprove only outputs it when instructed to do so with the switch --info, in which case it issues here the following messages:

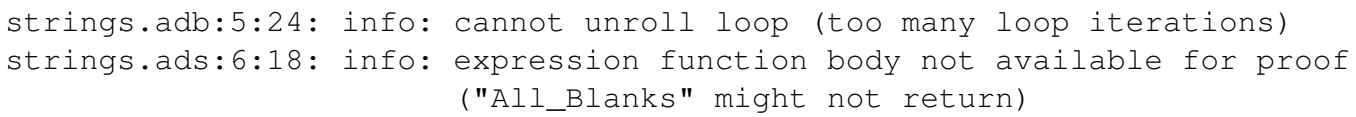


The first line informs the user that the loop in Erase could not be unrolled (hence it requires a loop invariant) because doing so would require too many loop iterations. Indeed, a string in SPARK is an array over the range of positive (32-bits) integers, a range much too large to unroll the loop.

The second line informs the user of another problem here: although function All_Blanks was defined as an expression function (a purely functional expression for a function, which can readily be translated into an axiom for proof), its defining expression cannot be used for interpreting the postcondition here. Indeed, All_Blanks is defined recursively, which makes it possible that it does not return on some inputs. In such cases, it would be unsound for GNATprove to treat its defining expression as an axiom in proof, which prevents using it here. There are multiple ways to solve this problem, either by providing a subprogram variant in order to prove termination, or by expressing All_Blanks differently without recursion.

Still in this category of probable cause, GNATprove can attempt to detect inconsistencies in specifications or code, by trying to prove that the logical context for a given branch in the specification or the program entails the False proposition. As this involves additional calls to automatic provers, hence has an impact on running time, this is only done when the user chooses to do so with the switch --proof-warnings.

\section{The Magician: Suggesting a Possible Fix}

The ultimate goal of interactivity is to suggest a possible fix to the user, in those (alas, few!) cases where it is possible, either because some information is clearly missing, or because a faulty pattern can be recognized. Our experience with SPARK has shown a few such cases where the analyzer just stops short of fixing the code itself. Let's add a loop invariant to the loop in Erase:

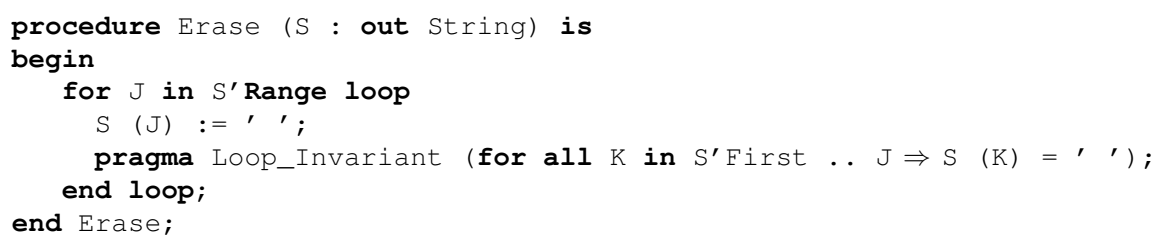

and reimplement All_Blanks without recursion as follows:

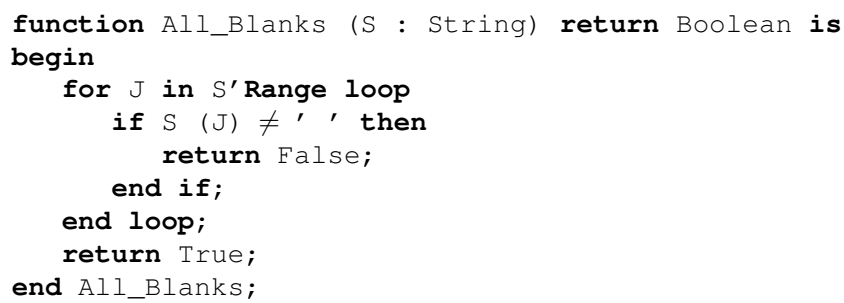

When running GNATprove on that code, it issues the following messages:

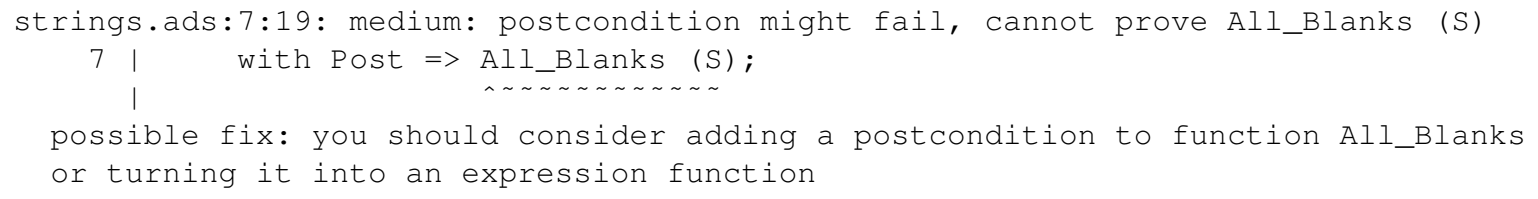

The poscondition of Erase still cannot be proved. GNATprove this time has a more precise suggestion for the solution, which is to add a postcondition to All_Blanks or to turn it into an expression 
function. Indeed, GNATprove handles differently regular functions, which may themselves contain imperative constructs like loops, and so-called expression functions, which can be readily interpreted in logical terms. Another option would be to add a postcondition to All_Blanks, which the message also mentions.

A similar case where GNATprove can suggest a precise fix to the user relates to the choice made in GNATprove to prove the absence of run-time errors inside preconditions independently from calling contexts. So if a subprogram has the expression (A and B) as a precondition, neither the evaluation of A nor the evaluation of B should lead to an error. In many cases though, evaluating B might require that A evaluates to True, and in such cases the precondition should be expressed using the shorthand connective "and then" as (A and then B). GNATprove detects cases where the user could have used "and then" instead of "and" in preconditions to protect against errors, and suggests this possible fix.

Another such case is the well-known misuse of a conditional inside an existential quantification, which beginners are almost certain to be bitten by at some point. When it finds such a syntactic construct (for some $\mathrm{X}=>$ (if $\mathrm{P}$ then $\mathrm{Q}$ )) which will evaluate to True whenever $\mathrm{P}$ is False, GNATprove issues a warning suggesting the likely fixes:

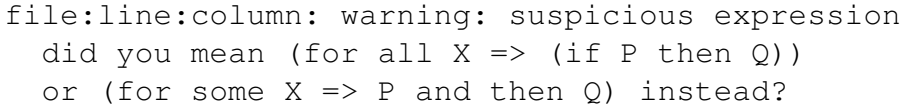

While this degree of feedback to the user is highly desirable, it is hard to produce in general, outside of the specific common cases described above.

\section{The Surgeon: Looking at the Innards}

This exploration would not be complete if we did not present the way for users to look at the innards of a Verification Condition, in cases where the analyzer did not present the information needed to understand the problem. Note however that the preferred means to investigate such unproved properties in SPARK is through so-called auto-active verification, where the user states intermediate properties through ghost code (assertions and lemmas). Let's define All_Blanks as an expression function whose body is the same universally quantified property that we wrote in the loop invariant:

function All_Blanks ( $S$ : String) return Boolean is

(for all $\mathrm{J}$ in $\mathrm{S}^{\prime}$ Range $\Rightarrow \mathrm{S}(\mathrm{J})={ }^{\prime}$, ) ;

When running GNATprove on that code, it proves the postcondition of Erase, but issues messages related to possible reads of uninitialized data (which were in fact issued on previous versions of the example), of the form:

file:line:column: "S" might not be initialized

The reason is that, by default, GNATprove checks correct data initialization by data flow analysis instead of proof, which is not sufficient here to prove that $\mathrm{s}$ is progressively initialized in the loop, which ends with $\mathrm{s}$ being completely initialized, and that only the initialized part of $\mathrm{s}$ is read in the loop invariant. The solution here is to indicate to GNATprove that we want it to treat $\mathrm{s}$ as partially initialized, and to use proof to demonstrate correct initialization before use:

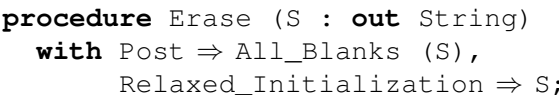


The effect is not immediately visible, as GNATprove keeps issuing messages about possible reads of uninitialized data. Ignoring for a moment that the User's Guide explains how to deal with such cases, we can try to understand by ourselves the underlying model used in proof to deal with initialization. Through a contextual menu, we can start manual proof on one of the unproved check, which opens multiple panels in the IDE [8]: a panel showing the proof tree (consisting in the tree of transformations and sub-goals), a panel displaying the current goal with names translated to reflect source code variable names (with hypotheses and conclusion), and a panel to enter commands to interact with the tool. After introducing quantified variables and hypotheses with the command split_vc, the goal looks like this:

goal def'vc : _attr_init (get2 $S_{-}$f) $=$True

We can display the definition of get 2 :

$>$ print get 2

function get2 ( $\left.f::^{\prime} a->{ }^{\prime} b\right)\left(x:{ }^{\prime} a\right):{ }^{\prime} b=f \backslash a x$

This is the application of a map representing the string to an index in order to get the corresponding element. Thus, the Verification Condition here looks at some attribute _attr_init representing the initialization status of this value, which should be the boolean True to denote that the value has been initialized. We can search for occurrences of __attr__init in the background theory encoding the program semantics and in the hypotheses encoding the subprogram execution, using the command search:

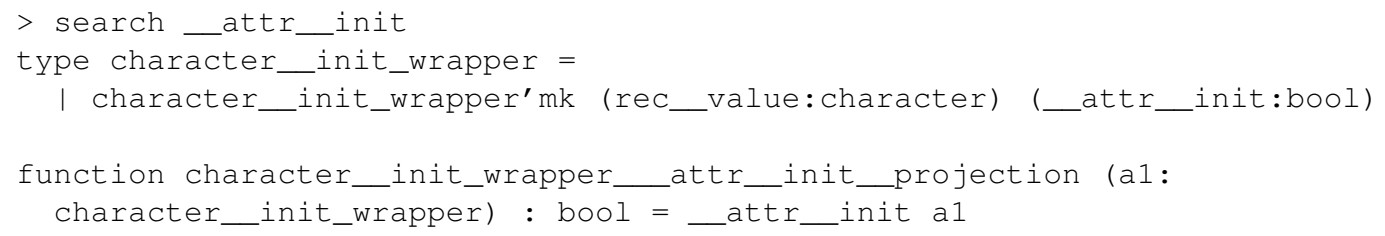

Here it returns elements of the background theory which allow to attach an initialization value to a character using type constructor character_init_wrapper'mk and to retrieve the corresponding value from the pair using function character_init_wrapper__attr__init__projection. And indeed the constructor is used to define what it means to initialize a character in function to_wrapper:

function to_wrapper (x:character) : character_init_wrapper =

character_init_wrapper'mk x True

which is used in one of the hypotheses to indicate that $\mathrm{S}(\mathrm{J})$ is initialized after the assignment inside the loop:

H1 : $S=\operatorname{set} 2 \mathrm{~S} 1 \mathrm{~J}$ (to_wrapper o)

So we only get that the element at the current index $J$ of the string $S$ is initialized, which is not sufficient here. What we need is to specify in a loop invariant that elements up to the current index have been initialized, using the attribute 'Initialized in SPARK:

pragma Loop_Invariant (for all $\mathrm{K}$ in $\mathrm{S}^{\prime}$ First . J $J \mathrm{~S}$ (K)'Initialized);

With that additional loop invariant, everything is proved about Erase, including the additional postcondition that $\mathrm{S}$ is fully initialized on return:

procedure Erase ( $S$ : out String)

with Post $\Rightarrow A l l \_B l a n k s$ (S) and then $S^{\prime}$ Initialized, Relaxed_Initialization $\Rightarrow S$;

As visible from this example, looking at the innards of proof requires some expertise which can only be acquired with time, to understand the mapping from source language constructs to logical encodings. The use of source variable names likes and $J$ in the Verification Condition presented to the user is a first step towards more systematic roundtrip translation into constructs at the source code level, to facilitate this understanding. 


\section{Related and Future Works}

Counterexamples are the main feature discussed in the context of interacting with program proof tools [6, 12]. Ideally, a counterexample captures in an understandable executable trace why a property cannot be proved, by exhibiting a consistent example where the property does not hold. In reality, after multiple person-year efforts to develop and improve counterexamples in the context of SPARK [7], there is still much to be desired here.

On the one hand, counterexamples are very valuable to beginners, to point at implicit assumptions they might have about the language or program semantics as well as misunderstandings about the way program proof in general or the specific SPARK proof tools work. This is particularly valuable for proving implicit properties of programs like absence of run-time errors, as programmers are not used to thinking about non-happy paths: How can a signed integer division overflow? How can a floating-point multiplication between positive values give zero as a result? On the other hand, counterexamples can be confusing when they are either spurious because they only reflect a limitation of the underlying provers (e.g. regarding non-linear arithmetic) or they do not represent a possible execution but only a limitation of the approach (e.g. related to the frame problem).

To reduce the possibility of confusion, we decided recently in SPARK to only enable counterexamples by default at higher levels of proof effort (when multiple provers are invoked for more than a few seconds per Verification Condition), so that counterexamples are generated in fewer cases and only in cases deemed difficult to prove. In parallel, we are working on using symbolic execution to verify the execution trace represented by a counterexample, in order to filter out spurious counterexamples and to better label the underlying error as a property violation or as a frame problem. Previous work on symbolic or concrete execution have shown benefits for better exploiting counterexamples [13, 10, 9, 5, 14].

Our experience with SPARK is that engineers have difficulties understanding the axiomatic semantics on which program proof is based. Their main mode of reasoning about programs is through wholeprogram operational semantics, which is supported by tools such as debuggers, fuzzers, profilers, etc. It is thus critical to provide tool feedback highlighting gaps between the two semantics which may explain why a property cannot be proved, such as the messages that we presented in the section 3 . This is an area where we will continue to look for ideas and improvements in the coming years.

\section{Conclusion}

Program proof is intrinsically an interactive effort between a human and a machine, as complete automation is not achievable. Thus, we are doomed to hit the so-called Left-Over Principle of automation [1, 2], which is that tasks that are not automated are precisely tasks where humans may not fare well either, because they are very infrequent or complex. More generally, the general understanding of the cooperation between the user and the tool in program proof could be improved, which may require the help of cognitive science [11]. And notations used to convey information to the tool could also benefit from the point of view of cognitive science [4]. Just looking at the specific issue of tool messages, the lessons learned from research on compiler error messages have direct implications for the design and implementation of program proof tools [3]. In this article, we presented the current state of such machine-to-human interactions in the SPARK technology, in the hope that it can trigger interesting human-to-human interactions in the community around proof tools. 


\section{References}

[1] https://www.kitchensoap.com/2012/09/21/a-mature-role-for-automation-part-i/ and https://www.kitchensoap.com/2013/08/20/a-mature-role-for-automation-part-ii/

[2] https://queue.acm.org/detail.cfm?id=2841313.

[3] Brett A. Becker, Paul Denny, Raymond Pettit, Durell Bouchard, Dennis J. Bouvier, Brian Harrington, Amir Kamil, Amey Karkare, Chris McDonald, Peter-Michael Osera, Janice L. Pearce \& James Prather (2019): Compiler Error Messages Considered Unhelpful: The Landscape of Text-Based Programming Error Message Research. In: Proceedings of the Working Group Reports on Innovation and Technology in Computer Science Education, ITiCSE-WGR '19, Association for Computing Machinery, New York, NY, USA, p. 177-210, doi 10.1145/3344429.3372508

[4] A. F. Blackwell, C. Britton, A. Cox, T. R. G. Green, C. Gurr, G. Kadoda, M. S. Kutar, M. Loomes, C. L. Nehaniv, M. Petre, C. Roast, C. Roe, A. Wong \& R. M. Young (2001): Cognitive Dimensions of Notations: Design Tools for Cognitive Technology. In Meurig Beynon, Chrystopher L. Nehaniv \& Kerstin Dautenhahn, editors: Cognitive Technology: Instruments of Mind, Springer Berlin Heidelberg, Berlin, Heidelberg, pp. 325-341, doi 10.1007/3-540-44617-6_31

[5] Maria Christakis, K. Rustan M. Leino, Peter Müller \& Valentin Wüstholz (2016): Integrated Environment for Diagnosing Verification Errors. In: 22nd International Conference on Tools and Algorithms for the Construction and Analysis of Systems (TACAS'16), pp. 424-441, doi 10.1007/978-3-662-49674-9_25

[6] David R. Cok (2010): Improved usability and performance of SMT solvers for debugging specifications. Int. Journal on Software Tools for Technology Transfer 12(6), pp. 467-481, doi 10.1007/s10009-010-0138-x

[7] Sylvain Dailler, David Hauzar, Claude Marché \& Yannick Moy (2018): Instrumenting a Weakest Precondition Calculus for Counterexample Generation. Journal of Logical and Algebraic Methods in Programming 99, pp. 97-113, doi 10.1016/j.jlamp.2018.05.003 Available at https://hal.inria.fr/hal-01802488.

[8] Sylvain Dailler, Claude Marché \& Yannick Moy (2018): Lightweight Interactive Proving inside an Automatic Program Verifier. In Paolo Masci, Rosemary Monahan \& Virgile Prevosto, editors: 4th Workshop on Formal Integrated Development Environment, Electronic Proceedings in Theoretical Computer Science 284, Open Publishing Association, Oxford, United Kingdom, doi:10.4204/EPTCS.284.1 Available athttps://hal.inria.fr/hal-01936302.

[9] Martin Hentschel, Reiner Hähnle \& Richard Bubel (2016): Deductive Software Verification - The KeY Book, chapter Debugging and Visualization, pp. 383-413. Springer, doi 10.1007/978-3-319-49812-6_11

[10] Bart Jacobs, Jan Smans, Pieter Philippaerts, Frédéric Vogels, Willem Penninckx \& Frank Piessens (2011): VeriFast: A Powerful, Sound, Predictable, Fast Verifier for C and Java. In Mihaela Gheorghiu Bobaru, Klaus Havelund, Gerard J. Holzmann \& Rajeev Joshi, editors: NASA Formal Methods, 6617, pp. 41-55, doi:10.1007/978-3-642-20398-5_4.

[11] G. Klien, D. D. Woods, J. M. Bradshaw, R. R. Hoffman \& P. J. Feltovich (2004): Ten challenges for making automation $a$ "team player" in joint human-agent activity. IEEE Intelligent Systems 19(6), pp. 91-95, doi 10.1109/MIS.2004.74

[12] Claire Le Goues, K. Rustan M. Leino \& Michal Moskal (2011): The Boogie Verification Debugger. In Gilles Barthe, Alberto Pardo \& Gerardo Schneider, editors: Software Engineering and Formal Methods - 9th International Conference, (SEFM), 7041, pp. 407-414, doi $10.1007 / 978-3-642-24690-6 \_28$

[13] Peter Müller \& Joseph N. Ruskiewicz (2011): Using Debuggers to Understand Failed Verification Attempts. In Michael J. Butler \& Wolfram Schulte, editors: 17th International Symposium on Formal Methods, 6664, pp. 73-87, doi $10.1007 / 978-3-642-21437-0 \_8$

[14] Guillaume Petiot, Nikolai Kosmatov, Bernard Botella, Alain Giorgetti \& Jacques Julliand (2016): Your Proof Fails? Testing Helps to Find the Reason. In: Tests and Proofs - 10th International Conference, 9762, pp. 130-150, doi: 10.1007/9783-319-41135-4_8. 